\title{
Hand-Finger Pose Estimation Using Inertial Sensors, Magnetic Sensors and a Magnet
}

\author{
Zhicheng Yang ${ }^{\circledR}$, Shenggang Yan ${ }^{\circledR}$, Bert-Jan F. van Beijnum ${ }^{\circledR}$, Member, IEEE, Bin Li $^{\circledR}$, \\ and Peter H. Veltink ${ }^{\circledR}$, Senior Member, IEEE
}

\begin{abstract}
Hand-finger motion tracking based on inertial and magnetic measurement units (IMMUs) has become a well-accepted method in many applications such as rehabilitation and virtual reality. However, existing systems require an IMMU on each segment of the whole hand and known length of each segment, which is hard to achieve for users. Therefore, we proposed a small sensor configuration with three IMMUs and a magnet to estimate poses of interested fingertips relative to the hand. The relative orientations were estimated by integrating relative angular velocity and fused with relative orientation estimation during time intervals when the whole hand moved or rotated as one object. The relative positions were estimated by using a magnetic dipole model of the magnet and prior estimated relative orientations. The performance has been demonstrated in multiple experiments. For whole hand rotation and functional grasping or writing experiments, the estimated errors of index fingertip position and orientation relative to the hand were $8.0 \sim 9.8 \mathrm{~mm}$ and $5.7^{\circ} \sim 11.2^{\circ}$. For index finger flexion and pinching, the estimated errors of index fingertip position and orientation relative to the hand were $16.2 \sim 25.2 \mathrm{~mm}$ and $20.6^{\circ} \sim 21.7^{\circ}$.
\end{abstract}

Index Terms-Magnetic localization, finger poses, IMMU, one magnetometer.

\section{INTRODUCTION}

$\mathbf{H}$ AND-FINGER motion tracking is a challenging problem and of wide interest in various applications, such as hand rehabilitation, enhancement of athletic performance and virtual reality [1]-[3]. The problem can be tackled by stateof-the-art systems, such as optical tracking systems (OTSs), electromagnetic tracking systems (EMTSs) or inertial tracking systems.

OTSs such as Vicon motion systems have high position accuracy and are often used as references. Typical drawbacks of OTSs are requirements of lab environment and line-of-sight

Manuscript received April 19, 2021; revised May 31, 2021; accepted May 31, 2021. Date of publication June 3, 2021; date of current version August 13, 2021. The work of Zhicheng Yang was supported by the China Scholarship Council (CSC). The associate editor coordinating the review of this article and approving it for publication was Dr. Marko Vauhkonen. (Corresponding author: Zhicheng Yang.)

This work involved human subjects or animals in its research. Approval of all ethical and experimental procedures and protocols was granted by the Ethics Committee EEMCS under Approval No. RP 2020-08.

Zhicheng Yang is with the School of Marine Science and Technology, Northwestern Polytechnical University, Xi'an 710072, China, and also with the Department of Biomedical Signals and Systems, Universiteity of Twente, 7522 NB Enschede, The Netherlands (e-mail: z.yang-1@utwente.nl).

Shenggang Yan and Bin Li are with the School of Marine Science and Technology, Northwestern Polytechnical University, Xi'an 710072, China.

Bert-Jan F. van Beijnum and Peter H. Veltink are with the Department of Biomedical Signals and Systems, Universiteity of Twente, 7522 NB Enschede, The Netherlands.

Digital Object Identifier 10.1109/JSEN.2021.3085993 problems etc [4], [5]. EMTSs such as Aurora systems have no line-of-sight occlusion. However, their drawbacks are that large volume emitters are required and they are susceptible to external ferromagnetic objects [6], [7].

What makes the use of the system based on inertial and magnetic sensors (IMMUs) especially attractive in comparison with the OTS and EMTS is that ambulatory assessments can be performed out of lab environments [8], [9]. Moreover, it can directly measure acceleration and angular velocity. Based on the raw data, orientation can be obtained by integrating the angular velocity provided with initial orientation. Owing to the drift errors, orientation cannot be estimated accurately for periods longer than dozens of seconds. Fortunately, drift issues can be solved by fusing the data from accelerometers and magnetometers, where accelerometers measure gravity and magnetometers measure geomagnetic field [10]-[12]. The performance of the compensation is related to non-gravitational accelerations and ferromagnetic disturbances. Compared with the orientation estimation, the position estimation based on inertial sensors seems to be more difficult, because extracted accelerations usually contain relatively larger errors and strapdown navigation, including double integration of the extracted accelerations makes the position estimation drift quite rapidly [13]. An alternative method for position estimation based on IMMUs is applying forward kinematics when the orientation and length of each segment are known. Several systems were designed to assess hand kinematics by using 
forward kinematics based on IMMUs, which were placed on each segment of the whole finger [14]-[16]. Subsequently, Salchow-Hommen et al. improved the robustness to magnetic disturbances by reducing magnetometers and simplifying 3DOF joint movements to 2DOF joint movements [17].

However, previous IMMU-based systems have several major drawbacks. Firstly, an IMMU on each finger segment is required, which are difficult to wear and calibrate since each IMMU needs to be well fixed and aligned with corresponding segment. Secondly, too much irrelevant information is estimated that increases the complexity of calculations. In many cases, we are only interested in the relative poses between fingertips and the dorsal side of the hand, but we need to estimate poses of all segments in order to use forward kinematics. Furthermore, errors of estimated positions accumulate along articulated chains because of the uncertainty of segment lengths and error parameters estimated by sensor to segment calibrations.

Compared with forward kinematics, a more robust solution to improve the position performance of IMMU-based systems is to fuse external position systems such as OTSs, ultrasound systems or EMTSs [18]-[20]. Weenk et al. proposed a method to estimate relative foot positions with inertial and ultrasonic sensors [21]. The position accuracy was improved compared with an IMMU-based system since ultrasound sensors provide distance information without drift. Roetenberg et al. proposed a method for ambulatory pose estimation by using IMMUs and orthogonal coils worn on the body [22]. Subsequently, Schepers et al. improved the hardware and algorithm [23]. Compared to the fusion of ultrasound systems, EMTSs provide position information instead of distance information, which is more suitable for the compensation of position drift. However, drawbacks still exist. Firstly, the body-worn EMTS is bulky, which hinder the subject's movements. Secondly, it consumes lots of energy, especially when the EMTS needs to cover a large tracking volume. To overcome those drawbacks, one potential method is to use a magnet as a passive emitter instead of active magnetic coils. Whereas, the magnet can only generate magnetic field along one axis instead of three axes like orthogonal coils. Furthermore, the magnetic field generated by the magnet is mixed with geomagnetic field, which cannot be easily extracted. Thus, the system requires more magnetometers as receivers to estimate poses of the magnet. Kortier et al. proposed a system including one magnet on the hand and four magnetometers on the trunk to estimate the pose of the hand relative to the trunk [24].

Inspired by Kortier et al.'s work, this paper presents a further step towards estimation of interested hand-finger poses using a small IMMU-based system and a magnet. The system contains three IMMUs placed on the index fingertip, thumb fingertip and dorsal side of the hand. No IMMU is attached to the intermediate segments. As fingertips are much smaller compared with the trunk, we proposed to only attach one magnetometer per fingertip instead of four. A new method with IMMUs and one magnetometer is proposed to estimate finger poses relative to the hand with IMMUs and one magnetometer, which is divided into two steps. Firstly, the relative orientations between the hand and fingertips are estimated based on inertial

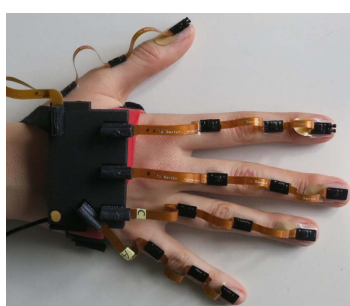

(a)

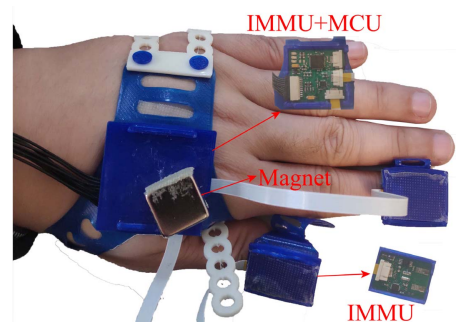

(b)
Fig. 1. (a) IMMU-based system from Salchow-Hommen et al.'s work [17], with an IMMU on each segment (b) The proposed small IMMU-based system, one IMMU on the tip of the thumb, one on the index fingertip and one on the dorsal side of the hand, with a magnet attached.

sensors [25]. Subsequently, relative orientations are combined with magnetometer outputs to estimate the relative positions between the hand and fingertips. The information that the hand and fingers often share approximately the same angular velocity or acceleration if the whole hand is moved or rotated is exploited to compensate the relative direction drift without magnetometers. The contribution of this paper is proposing a small IMMU-based system to estimate relative poses between the hand and fingertips of interest, without known segment lengths or requirement of forward kinematics.

\section{Methods}

In order to estimate the fingertip poses relative to the hand, a small IMMU-based system and corresponding algorithms were designed.

\section{A. System Design}

A traditional system from previous research is shown in Fig 1a. The proposed system is shown in Fig 1b. Two IMMUs are fixed on the thumb and index fingertip respectively. One IMMU is fixed on the dorsal side of the hand with one magnet attached above. It looks like our system is larger than the system shown in Fig 1a. However, we used the same sensor MPU9250 on finger segments. The reasons why it looks larger are: firstly, our system reserved an interface and peripheral circuits for pressure sensor on fingertip, which we may fuse in the future work. However, it occupies a large space. Secondly, in order to make the system easier to wear, we made a $3 \mathrm{D}$ printed coat, which also makes our system looks larger.

Compared with previous systems, our system concentrates on the movements of thumb and index fingertips. Thus, IMMUs attached to the middle and proximal phalanx are not included. As a consequence, forward kinematics cannot be used to estimate the relative positions anymore. The magnet on the dorsal side of the hand is used as a passive magnetic source to estimate finger positions relative to the hand. However, the introduction of the magnet disturbs the geomagnetic field measured by magnetometers. Therefore, the magnetometer cannot be used to compensate the direction drift. Instead of exploiting the geomagnetic field, we exploit the information collected when the hand moves as a whole object to compensate the direction drift.

The process of estimating the $6 \mathrm{D}$ relative poses between the hand and fingertips is shown in Fig 2. The relative orientation is estimated first, which is a necessary condition to estimate relative position. 


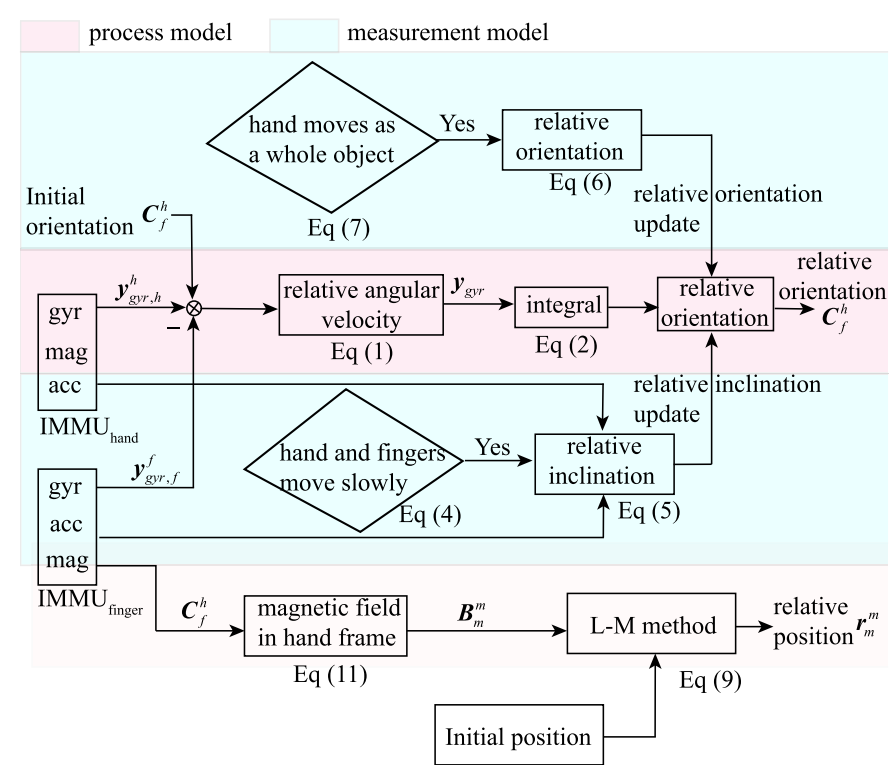

Fig. 2. Algorithm for the estimating fingertip poses relative to the hand with IMMUs and a magnet.

\section{B. Estimation of Relative Orientations With Inertial Sensors}

As shown in Fig 2, the relative orientation between the hand and fingertips is obtained by integrating relative angular velocity, and compensated by the relative inclination and the information collected when the hand moves as a whole object [10].

1) Estimation of Relative Orientation Change: The relative angular velocity between the hand and fingertips can be obtained as

$$
\boldsymbol{y}_{g y r}=\boldsymbol{y}_{g y r, h}^{h}-\boldsymbol{C}_{f}^{h} \boldsymbol{y}_{g y r, f}^{f}
$$

where $\boldsymbol{y}_{g y r, h}^{h}$ and $\boldsymbol{y}_{g y r, f}^{f}$ are gyroscope outputs on the hand and fingertips in their own frames. $\boldsymbol{C}_{f}^{h}$ is the relative orientation between the hand and fingertips based on rotation matrix, which can be updated with relative angular velocity $\boldsymbol{y}_{g y r}$ :

$$
\dot{\boldsymbol{C}}_{f}^{h}=\boldsymbol{C}_{f}^{h}\left[\boldsymbol{y}_{g y r} \times\right]
$$

where $\left[\boldsymbol{y}_{\text {gyr }} \times\right]$ is

$$
\left[\boldsymbol{y}_{g y r} \times\right]=\left[\begin{array}{ccc}
0 & -y_{g y r}, z & y_{g y r, y} \\
y_{g y r, z} & 0 & -y_{g y r, x} \\
-y_{g y r, y} & y_{g y r, x} & 0
\end{array}\right]
$$

$y_{g y r, x}, y_{g y r, y}, y_{g y r, z}$ are three components of $\boldsymbol{y}_{g y r}$. The update of orientation $\boldsymbol{C}_{f}^{h}$ based on Eq 2 may introduce drift. Therefore, this orientation estimate needs to be fused with some other information about relative orientation that does not drift.

2) Estimation of Relative Inclination Using Accelerometers: When the hand and fingers move or rotate slowly, the nongravitational acceleration is negligible compared with the gravity.

$$
||\left|\boldsymbol{y}_{a c c, h}^{h}\left\|_{2}-g\left|<\varepsilon_{a},\right||| \boldsymbol{y}_{a c c, f}^{f}\right\|_{2}-g\right|<\varepsilon_{a}
$$

where $\boldsymbol{y}_{a c c, h}^{h}$ and $\boldsymbol{y}_{a c c, f}^{f}$ are accelerometer outputs in their own frames. $g$ is the local gravity. $\varepsilon_{a}$ guarantees the non-gravitational acceleration is not too large when the relative inclination is estimated. The relation of accelerometers between the hand and fingers can be approximated as

$$
\boldsymbol{y}_{a c c, h}^{h} \approx \boldsymbol{C}_{f}^{h} \boldsymbol{y}_{a c c, f}^{f}
$$

3) Relative Orientation Estimation Under the Condition That the Hand and Fingers Accelerate or Rotate Approximately as One Object: Eq (3) cannot fully compensate the relative orientation drift caused by the time integral of relative angular velocity. Fortunately, the hand and fingers often move as a whole object during daily tasks. Thus, they frequently share approximately the same acceleration and angular velocity.

$$
\left\{\begin{array}{l}
\boldsymbol{y}_{g y r, h}^{h} \approx \boldsymbol{C}_{f}^{h} \boldsymbol{y}_{g y r, f}^{f} \\
\boldsymbol{y}_{a c c, h}^{h} \approx \boldsymbol{C}_{f}^{h} \boldsymbol{y}_{a c c, f}^{f}
\end{array}\right.
$$

In order to determine when the hand and fingers moves as a whole object, a decision algorithm was designed

$$
\left\{\begin{array}{c}
\left|\left\|\boldsymbol{y}_{g y r, h}^{h}\right\|_{2}-\left\|\boldsymbol{y}_{g y r, i}^{i}\right\|_{2}\right|<\varepsilon_{b}, \mid\left\|\boldsymbol{y}_{a c c, h}^{h}\right\|_{2} \\
-\left\|\boldsymbol{y}_{a c c, i}^{i}\right\|_{2} \mid<\varepsilon_{c} \\
\left\|\boldsymbol{y}_{g y r, h}^{h}\right\|_{2}>\varepsilon_{d},\left\|\boldsymbol{y}_{g y r, i}^{i}\right\|_{2}>\varepsilon_{d}
\end{array}\right.
$$

where $\varepsilon_{b}$ and $\varepsilon_{c}$ guarantee the hand and fingers share approximately the same acceleration and angular velocity. $\varepsilon_{d}$ guarantees the hand is rotating rather than stationary. In this way, $\boldsymbol{C}_{f}^{h}$ can be estimated based on Eq (4). $\varepsilon_{a, b, c, d}$ are chosen according to the desired movement complexity [25]. In this experiment, the tuning parameters $\varepsilon_{a, b, c, d}$ were set as:

$$
\left\{\begin{array}{lll}
\varepsilon_{a}=0.05 g, & \varepsilon_{b}=0.1\left\|\boldsymbol{y}_{\text {gyr }, h}^{h}\right\|_{2} \\
\varepsilon_{c}=0.1\left\|\boldsymbol{y}_{a c c, i}^{i}\right\|_{2}, & \varepsilon_{d}=0.4 \mathrm{rad} / \mathrm{s}
\end{array}\right.
$$

\section{Estimation of Relative Positions With Known Orientations and a Magnetometer}

When a ferromagnetic object is far from the magnetometer, more than 2.5 times of its length, the object can be approximated as a dipole [26], [27]. The magnetic field generated by a dipole in its own frame is

$$
\boldsymbol{B}_{m}^{m}=f\left(\boldsymbol{r}_{m}^{m}\right)=\frac{\mu_{0}}{4 \pi}\left(\frac{3\left(r_{m}^{m}\right)^{T} \boldsymbol{M}_{m}^{m} \boldsymbol{r}_{m}^{m}}{\left(r_{m}^{m}\right)^{5}}-\frac{\boldsymbol{M}_{m}^{m}}{\left(r_{m}^{m}\right)^{3}}\right)
$$

where $\mu_{0}$ is the permeability of free space, $\boldsymbol{M}_{m}^{m}$ is the magnetic moment in magnet frame, $\boldsymbol{r}_{m}^{m}$ is the position in magnet frame and $r_{m}^{m}$ is the corresponding distance. $\left(\boldsymbol{r}_{m}^{m}\right)^{T}$ is the transpose of $\boldsymbol{r}_{m}^{m}$. The magnetometer output on the fingertip is

$$
\boldsymbol{y}_{\text {mag }}^{s}=\boldsymbol{C}_{f}^{s}\left(\boldsymbol{C}_{f}^{h}\right)^{-1} \boldsymbol{C}_{m}^{h} \boldsymbol{B}_{m}^{m}+\boldsymbol{B}_{e}^{s}+\boldsymbol{n}_{B}
$$

where $\boldsymbol{B}_{e}^{S}$ is the geomagnetic field in magnetometer frame. $\boldsymbol{n}_{B}$ is the measurement error. $\boldsymbol{C}_{m}^{h}$ is the rotation matrix between the magnet and hand. $\boldsymbol{C}_{f}^{s}$ is the rotation matrix between the magnetometer and fingertips. $\boldsymbol{C}_{m}^{h}$ and $\boldsymbol{C}_{f}^{s}$ can be obtained prior to measurements through calibration procedures. $\boldsymbol{C}_{f}^{h}$ can be obtained as described previously in subsection B on page 2. When the sum of $\boldsymbol{B}_{e}^{s}$ and $\boldsymbol{n}_{B}$ are 
much smaller than $\boldsymbol{B}_{m}^{m}$ (depending on the required position accuracy), Eq (10) can be simplified as

$$
\boldsymbol{B}_{m}^{m} \approx\left(\boldsymbol{C}_{m}^{h}\right)^{-1}\left(\boldsymbol{C}_{f}^{h}\right)\left(\boldsymbol{C}_{f}^{s}\right)^{-1} \boldsymbol{y}_{\text {mag }}^{s}
$$

$\boldsymbol{r}_{m}^{m}$ can be estimated with Levenberg-Marquardt (L-M) method based on Eq (11). However, two possible solutions will be estimated since

$$
f\left(\boldsymbol{r}_{m}^{m}\right)=f\left(-\boldsymbol{r}_{m}^{m}\right)
$$

The ghost solution can be eliminated by a good initial value or the general range of the finger movement.

\section{EXPERIMENTAL Methods}

\section{A. Experiment Setups}

MPU9250 (InvenSense) was chosen as the IMMU. The magnetometer and accelerometer were sampled at $100 \mathrm{~Hz}$. The gyroscope was sampled at $200 \mathrm{~Hz}$. All the data was transmitted to a PC through a USB connection. A $1.1 \mathrm{~cm} \times 1.1 \mathrm{~cm} \times$ $1.1 \mathrm{~cm}$ magnet with the magnetic moment of $3.2 \mathrm{~A} \cdot \mathrm{m}^{2}$ was chosen as a passive magnetic source based on two criteria: (1) the magnetic field generated by the magnet should be much stronger than the geomagnetic field in the movement range. (2) The length of the magnet should be much smaller than the estimated distance. The Vicon system with 8 cameras was used to provide the position and orientation reference. Two clusters of markers were attached to the fingertips of thumb and index finger. A cluster of markers was attached to the surface of the magnet. Each cluster contained three markers. The sample frequency of Vicon system was $100 \mathrm{~Hz}$.

\section{B. References From the Vicon System}

The position reference was obtained from the measurement of one marker. The orientation reference was estimated from a cluster of markers. For each cluster, we obtain two vectors $\boldsymbol{r}_{1}, \boldsymbol{r}_{2}$ spanning a configuration of three markers. Then the orientation of the marker frame can be calculated as:

$$
\boldsymbol{C}_{M}^{g}=\left[\begin{array}{lll}
\frac{\boldsymbol{n}_{\boldsymbol{r}_{2}} \times \boldsymbol{n}_{\boldsymbol{r}_{3}}}{\left|\boldsymbol{n}_{\boldsymbol{r}_{2}} \times \boldsymbol{n}_{\boldsymbol{r}_{3}}\right|} & \boldsymbol{n}_{\boldsymbol{r}_{2}} & \boldsymbol{n}_{\boldsymbol{r}_{3}}
\end{array}\right]
$$

where $\boldsymbol{n}_{\boldsymbol{r}_{3}}=\boldsymbol{n}_{\boldsymbol{r}_{1}} \times \boldsymbol{n}_{\boldsymbol{r}_{2}} /\left|\boldsymbol{n}_{\boldsymbol{r}_{1}} \times \boldsymbol{n}_{\boldsymbol{r}_{2}}\right| . \boldsymbol{n}_{\boldsymbol{r}_{1}}$ and $\boldsymbol{n}_{\boldsymbol{r}_{2}}$ are normalized vectors corresponding to $\boldsymbol{r}_{1}$ and $\boldsymbol{r}_{2}$. $\boldsymbol{C}_{M}^{g}$ is the orientation of optical markers in the global frame of the OTS. The global frame of OTS was determined by the calibration procedures of the OTS. Through the rotation matrix between the marker frame and sensor frame, the orientation assessed by the OTS can be easily transferred to the sensor frame.

\section{Synchronization and Alignment Between the IMMU and OTS}

The IMMU and Vicon system were two independent systems. They needed to be synchronized and aligned because the Vicon system provided the orientation and position reference for IMMUs. Angular velocities obtained from the IMMU-based system and Vicon system were used to achieve this goal.

For the IMMU system, angular velocities could be measured by the gyroscope. For the Vicon system, the orientation can

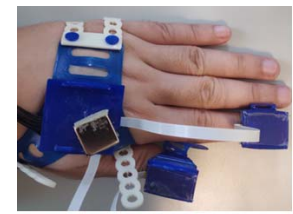

(a)

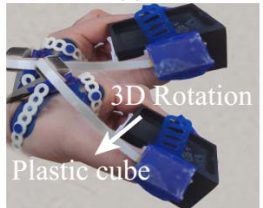

(d)

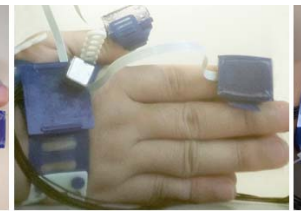

(b)

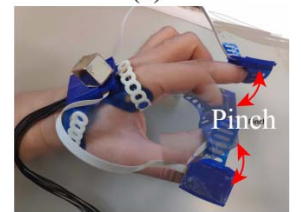

(e)

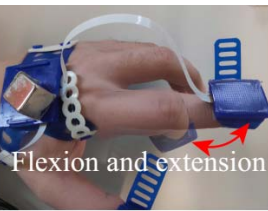

(c)

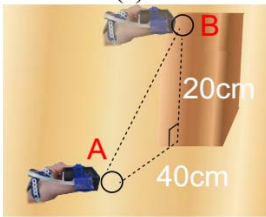

(f)
Fig. 3. Illustration of the movements in the experiments. (a) Hand flat on the table. (b) Hand vertically on the table. (c) Index finger flexion and extension. (d) Hold a plastic cube and rotate in 3D space, while the relative orientation between the hand and fingers remains unchanged. (e) Pinch movement. (f) Move a plastic cube from point A to point $B$. The horizontal and vertical distance between position $A$ and $B$ are $40 \mathrm{~cm}$ and $20 \mathrm{~cm}$, respectively.

be obtained based on the cluster of markers. Subsequently, angular velocities could be estimated. We maximized the correlations of both norms to achieve the synchronization. The alignment can be achieved by the following equation

$$
\boldsymbol{C}_{M}^{s}=\operatorname{argmin}\left(\sum_{k=1}^{N}\left\|\left(\hat{\boldsymbol{\omega}}^{s}\right)_{k}-\boldsymbol{C}_{M}^{s}\left(\hat{\boldsymbol{\omega}}^{M}\right)_{k}\right\|_{2}^{2}\right)
$$

where $\hat{\boldsymbol{\omega}}^{M}$ is the angular velocity in the marker frame. $\hat{\boldsymbol{\omega}}^{s}$ is the angular velocity in the gyroscope sensor frame. $C_{M}^{S}$ is the rotation matrix from the marker frame to the sensor frame.

\section{Protocols}

The performance of the proposed method was verified by different movements from 8 participants. The protocol was approved by ethics committee of Faculty of Electrical Engineering, Mathematics and Computer Science of the University of Twente.

1) Sensor to Segment Calibration: Before the measurement, IMMUs need to be aligned to segments of the hand. Gyroscopes and accelerometers were exploited to realize such calibration. The procedures are described as follows, more details can be found in related research [14].

(1) Put the hand flat on the table and keep static for $20 \mathrm{~s}$, with the back of the hand facing upward, as shown in Fig 3a;

(2) Put two hands together on the table vertically and hold on for $20 \mathrm{~s}$, as shown in Fig 3b;

(3) Flex and extend the index finger repeatedly for $20 \mathrm{~s}$, as shown in Fig 3c;

2) Initial Orientation Estimation: Lay the palm and fingers flat, and then rotate the hand as a whole object along the middle finger for $10 \mathrm{~s}-$ at the rate of 3 times per second.

Experiment 1-3D random rotation without changing relative orientations between the hand and fingertips

Hold a plastic cube while not changing the relative orientations between the hand and fingers, and then rotate the cube randomly in 3D space, as shown in Fig 3d.

\section{Experiment 2-Grasping tasks}

(1) Put the palm flat on the table near initial position A for $10 \mathrm{~s}$; 
(2) Grasp the plastic object with tips of the index finger and thumb from the position $\mathrm{A}$ and put it to the position B, and then move the hand to the initial position $\mathrm{A}$, as shown in Fig 3f. The horizontal and vertical distances between position A and B are $40 \mathrm{~cm}$ and $20 \mathrm{~cm}$ respectively;

\section{Experiment 3-Writing tasks}

Put the palm flat on the table, and then pick up a pen and write for $20 \mathrm{~s}$.

\section{Experiment 4-Flexion and extension}

Stretch the arm, and then flex and extend the index finger for $20 \mathrm{~s}-$ at the rate of 3 times per second;

\section{Experiment 5-Pinch}

Stretch the arm, and then perform a pinch movement with the thumb and the index finger for $20 \mathrm{~s}$, at the rate of 3 times per second, as shown in Fig 3e.

\section{Results}

Fig 4 shows the 6D pose tracking results of the grasping experiment. It can be seen there is no obvious drift error both for relative orientations and positions. The average errors for estimations of relative positions and orientations are $10.2 \mathrm{~mm}$ and $9.6^{\circ}$ respectively.

Fig 5 shows the $6 \mathrm{D}$ pose tracking results of the flexion experiment. It can be seen there are obvious drift errors either for relative orientations or positions. The average errors for estimations of relative positions and orientations are $19.3 \mathrm{~mm}$ and $16.4^{\circ}$ respectively. Compared with the grasping experiment, the flexion experiment did not include the condition that the hand moved as a whole object. Therefore, drift in relative orientation estimation could not be avoid.

Fig 6 shows the estimated errors of several movements. Estimated position and orientation errors for the grasping, writing and rotating movements are smaller. During these movements, we had moments when the hand moved as a whole object and obtained enough information to compensate drift errors.

\section{DISCUSSION}

We proposed and evaluated a small setup including 3 IMMUs and a magnet to estimate the poses of interested fingertips relative to the hand. Several studies performed movement experiments on human objects and compared results with an optical tracking system. In Kortier et al.'s research where the finger flexion and circular were performed, the estimated position error between the hand and index fingertip was $5.7 \sim 12.4 \mathrm{~mm}$, and the estimated orientation error was less than $5.0^{\circ} \sim 8.4^{\circ}$ for each segment of the hand during flexion and extension movements. During the repeatability tests, the orientation error for a single posture was less than $2^{\circ}$.

In Salchow-Hömmen et al.'s research where finger flexion and the combination movement of abduction and flexion were performed, the estimated position error between the hand and index fingertip was $5 \sim 21 \mathrm{~mm}$, and the estimated orientation error was 5 16 deg for each segment. Both studies applied an IMMU on each segment of fingers and a kinematic model of the hand.

In comparison, the precision of the proposed method depends on the complexities of movements. For rotating,
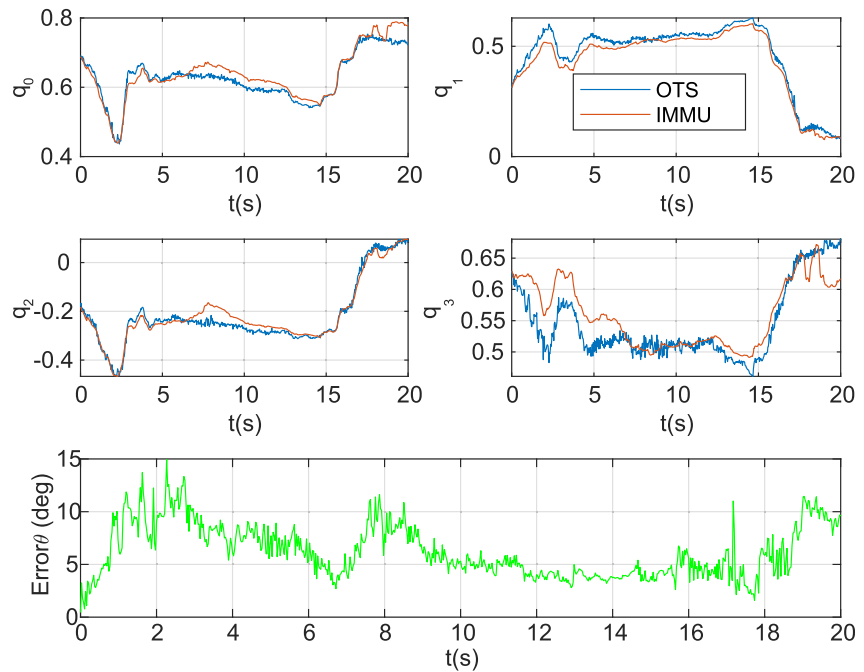

(a)
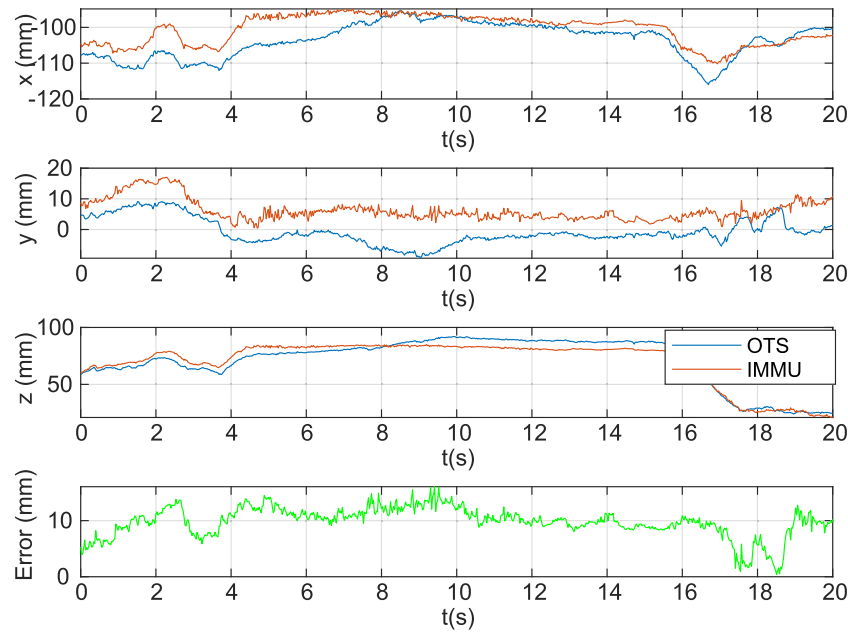

(b)

Fig. 4. 6D pose tracking results of the grasping experiment. (a) Fingertip orientation relative to the hand in the grasping experiment. (b) Fingertip position relative to the hand in the grasping experiment.

TABLE I

Comparison of the PERFormance of the PROPOSEd METHOd With Others'

\begin{tabular}{|c|c|c|c|c|}
\hline Items & IMMU & Magnet & Position error & $\begin{array}{l}\text { Orientation } \\
\text { error }\end{array}$ \\
\hline $\begin{array}{l}\text { Salchow-Hömmen } \\
\text { et al. }{ }^{[1]}\end{array}$ & 16 & No & $5 \sim 21 \mathrm{~mm}$ & $5^{\circ} \sim 16^{\circ}$ \\
\hline Kortier et al. ${ }^{[1]}$ & 18 & No & $5.7 \sim 12.4 \mathrm{~mm}$ & $5.0^{\circ} \sim 8.4^{\circ}$ \\
\hline The proposed method ${ }^{2}$ & 2] 3 & Yes & $\begin{array}{c}8.0 \sim 9.8 \mathrm{~mm} \\
16.2 \sim 25.2 \mathrm{~mm}\end{array}$ & $\begin{aligned} & 5.7^{\circ} \sim 11.2^{\circ} \\
& 20.6^{\circ} \sim 21.7^{\circ}\end{aligned}$ \\
\hline
\end{tabular}

${ }^{1}$ For Salchow-Hömmen et al.'s and Kortier et al.'s work, the orientation error was refer to the error between two segments, not between the hand and fingertips.

${ }^{2}$ For the proposed method, the performance was related to the quality of the movements. For the first row, there was an opportunity that hand moved as a whole object, which could be used to update the orientation, while there was not such opportunity for the result in the second row.

grasping and writing movements, the orientation error for each segment was not estimated, but the estimated orientation error between the hand and index finger was $5.7^{\circ} \sim 11.2^{\circ}$. The estimated position error between the hand and index finger was $8.0 \sim 9.8 \mathrm{~mm}$. The estimated position and orientation 

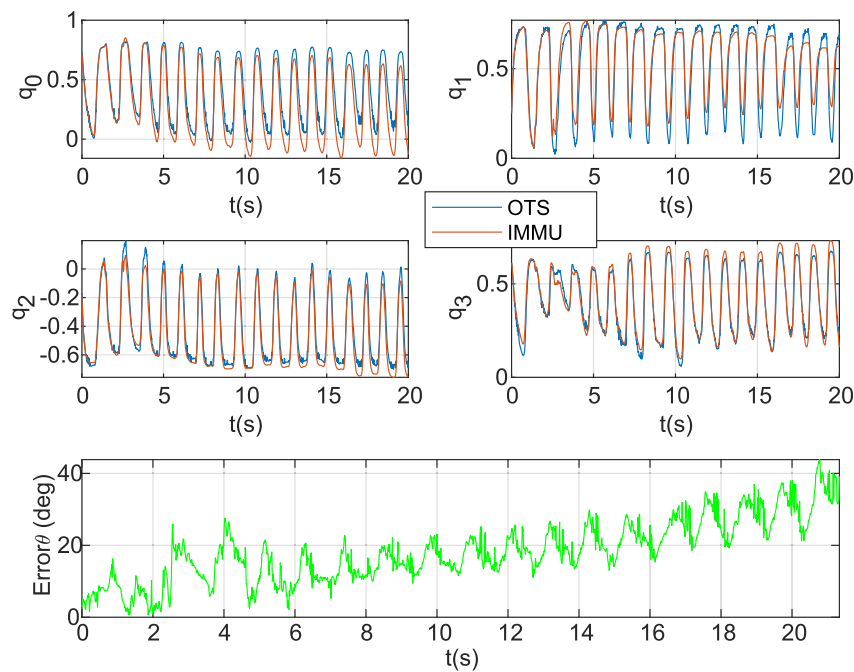

(a)
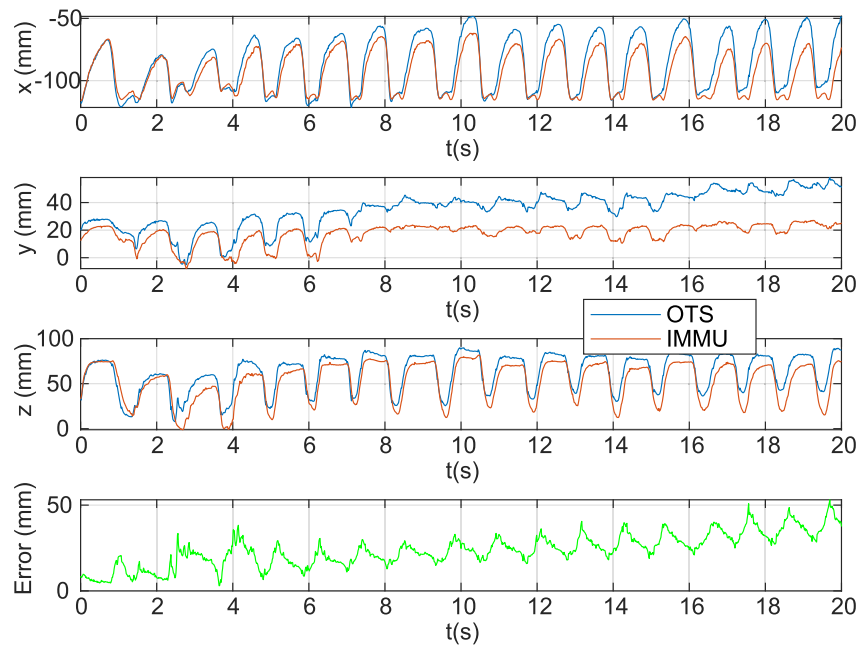

(b)

Fig. 5. 6D pose tracking results of the flexion experiment. (a) Position tracking in the flexion experiment. (b) Orientation tracking in the flexion experiment. $6 \mathrm{D}$ pose tracking results in experiments of index finger flexion and extension. (a) Fingertip orientation relative to the hand orientation in experiments of index finger flexion and extension. (b) Fingertip position relative to the hand position in experiments of index finger flexion and extension.
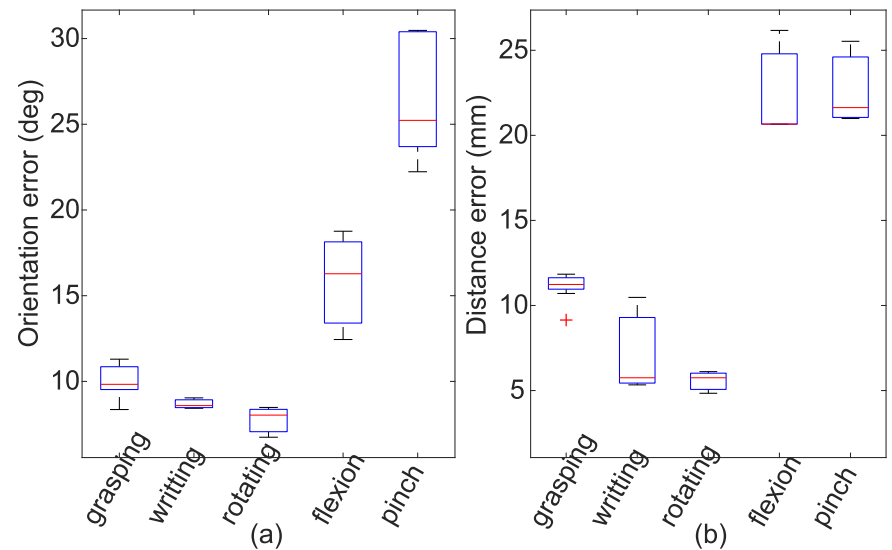

Fig. 6. Median pose errors from 8 participants during different movements. (a) Relative Orientation error with different movements. (b) Relative position error with different movements.

errors are comparable to Kortier et al.'s and Salchow-Hömmen et al.'s results [14], [17]. For the flexion and pinch movements, the orientation and position errors between the hand and index fingers were $20.6^{\circ}$ $\sim 21.7^{\circ}$ and $16.2 \sim 25.2 \mathrm{~mm}$ (see Fig 6), which are larger than Kortier et al.'s and Salchow-Hömmen et al.'s results [14], [17]. Compared with Kortier et al.'s and Salchow-Hömmen et al.'s systems, the advantages of the proposed method are that IMMUs attached to the intermediate segments and the lengths of finger segments are not required.

For the estimation of relative orientations, the accuracy depends on the complexity of movements. The estimation may have large drift if there is no chance that the whole hand moves or rotates as a whole object. However, the drift can be reduced by applying smoothing techniques, such as Rauch-TungStriebel (RTS) smoother, since for the orientation estimation at $t_{k}$, the information during the whole period can be exploited with smoothing technique, while the EKF can only exploit information between $0 \sim t_{k}$.

For the estimation of relative positions, there are several factors that affect the results. Firstly, the accuracy of relative position estimations is related to the relative orientation estimations, since the estimated relative orientations are used as input variables to calculate relative positions. Secondly, the magnetic field generated by the magnet is assumed to be much larger than the geomagnetic field. The estimation errors can be large when the assumption is not true. Thirdly, the model of the magnet is simplified as a dipole model which may cause errors, especially when the distance between the magnetometer and magnet is less than 2.5 times of the magnet's length. Fourthly, disturbances caused by ferromagnetic objects can also affect the accuracy of relative positions. The impact depends on the intensity of the ferromagnetic disturbances. Besides, the optical markers did not coincide with IMMUs. In our experiment, the relative position between them was roughly estimated from the experimental photos, thus may contained errors. There are small offset errors in Fig 4 and 5, which may be caused by the non-coincidence of the marker and IMMU.

The geomagnetic field is assumed as a disturbance and could not be estimated because we only have one magnetometer on the fingertip. If the geomagnetic field can be estimated with more magnetometers, the position accuracy can be improved. Moreover, if we have a magnetometer array with four magnetometers, the relative orientations between the magnet and fingertips can be estimated by using magnetometers alone. In that case, the update of relative orientations does not depend on the complexity of the movements anymore. Then we can obtain good estimations during a long period no matter what type of the movement is. When adding more magnetometers is feasible, the size and strength of magnet can be reduced. Nevertheless, the distance between magnetometers should be large enough that the differential magnetometer field measured by the magnetometer array is much larger than the noise. When adding new magnetometers is not achievable, such as the configuration in our approach, the gyroscope and accelerometer combined with magnetometer can be used to reduce the impact of the geomagnetic field. As shown in Fig 7 , we obtain the inclination $\boldsymbol{C}_{g}^{f}$ of the fingertip with the gyroscope and accelerometer. Only the z-component of the 


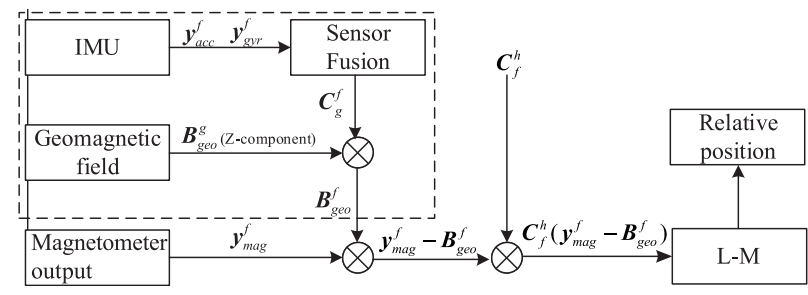

Fig. 7. Illustration of the position accuracy improvement by compensating the z-axis component of geomagnetic field. $\boldsymbol{B}_{\text {geo }}^{g}$ is the z-axis component of geomagnetic field.

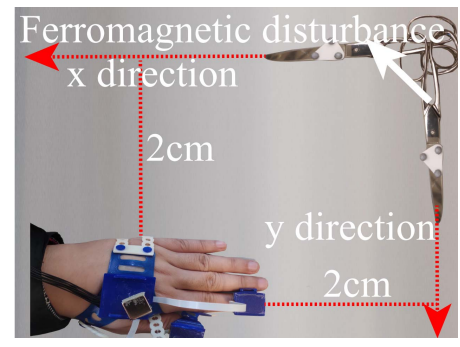

Fig. 8. Experiment of the influence of the ferromagnetic object. The scissors were chosen as the ferromagnetic object, which moved along two directions. During the experiment, the hand kept static.

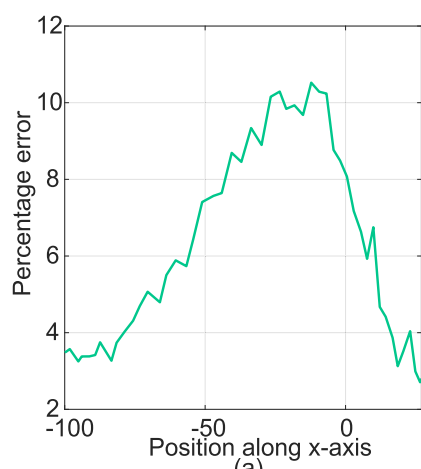

(a)

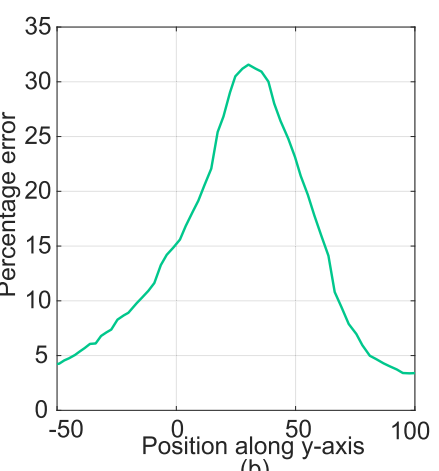

(b)
Fig. 9. Relative position errors between the hand and the index finger in the presence of ferromagnetic disturbances. (a) and (b) show the distance error with trajectories of the ferromagnetic object along $\mathrm{x}$-axis and $y$-axis. The moving direction can be seen in Fig $9 \mathrm{~h}$.

geomagnetic field in the sensor frame can be compensated by the fused inclination $\boldsymbol{C}_{g}^{f}$. In this way, the z-component of the geomagnetic field disturbance is removed. The performance of this method depends on the location on earth since the z-component of the geomagnetic field varies at different locations. Except the geomagnetic disturbance, the magnetic surroundings may influence the performance of the proposed method. The estimation error depends on the magnetic surroundings' strength, direction etc. One experiment for the test of magnetic surroundings is shown in appendix I.

In this experiment, only 8 healthy participants involved to do individual movements. Next step, we are going to apply the system to stroke patients and evaluate its performance under longer monitoring with sequential movements. As the strength of the magnet is much stronger than the geomagnetic field, it may cause unwanted forces if it is used near iron objects, such as iron tables or chairs.

\section{CONCLUSION}

A small configuration based on IMMUs and a magnet was proposed to estimate fingertip poses of interest relative to the hand. The purpose of this approach is to avoid using prior information on the length of each segment and use as few IMMUs as possible. The fingertip orientations relative to the hand were firstly estimated by integrating the relative angular velocity. Subsequently, the fingertip position drift can be reduced by estimating fingertip orientations relative to the hand when the hand and fingers accelerate or rotate as a whole object, using the accelerometer and gyroscope. Then the relative positions were estimated using the relative orientations and magnetometer information. For rotating, grasping and writing movements, the estimated relative position and orientation errors between the hand and fingertips were $8.0 \sim 9.8 \mathrm{~mm}$ and $5.7^{\circ} \sim 11.2^{\circ}$ respectively. For the flexion and pinch movements, the relative position and orientation errors between the hand and fingertips were $16.2 \sim 25.2 \mathrm{~mm}$ and $20.6^{\circ} \sim 21.7^{\circ}$ respectively. In conclusion, the proposed method is a promising approach for the hand-finger motion tracking with comparable performance as previous methods that applied the kinematic model of the fingers and additional IMMUs on intermediate finger segments.

\section{Appendix A \\ MAgnetic Disturbance TEST}

The experiment procedure is shown in Fig 8, the pair of scissors was chosen as the ferromagnetic object and moved along two directions during the experiment. When the pair of scissors moved approximately along the $\mathrm{x}$-axis of the hand frame, the largest estimated distance error was $10.6 \%$, as shown in Fig 8a. When the pair of scissors moved approximately along the y-axis, the largest distance error was 32.3\%, as shown in Fig 8b. The distance error is related to the direction of the pair of scissors.

\section{ACKNOWLEDGMENT}

The authors would like to thank the Roessingh Research and Development (Enschede, The Netherlands) for sharing the gait laboratory and the Lab Manager, Leendert Schaake, for assistance using the optical system and processing of data. They thank A. Droog and G.J.W. Wolterink from the Biomedical Signals and Systems Research Group of the University of Twente, for providing the inertial sensor setup and the 3-D printed housing for inertial sensors.

\section{REFERENCES}

[1] C. D. Metcalf et al., "Markerless motion capture and measurement of hand kinematics: Validation and application to home-based upper limb rehabilitation," IEEE Trans. Biomed. Eng., vol. 60, no. 8, pp. 2184-2192, Aug. 2013.

[2] N. J. Seo, J. A. Kumar, P. Hur, V. Crocher, B. Motawar, and K. Lakshminarayanan, "Usability evaluation of low-cost virtual reality hand and arm rehabilitation games," J. Rehabil. Res. Develop., vol. 53, no. 3, p. 321, 2016

[3] J. Liu, P. Carr, R. T. Collins, and Y. Liu, "Tracking sports players with context-conditioned motion models," in Proc. IEEE Conf. Comput. Vis. Pattern Recognit., Jun. 2013, pp. 1830-1837.

[4] A. Pfister, A. M. West, S. Bronner, and J. A. Noah, "Comparative abilities of microsoft Kinect and vicon 3D motion capture for gait analysis," J. Med. Eng. Technol., vol. 38, no. 5, pp. 274-280, Jul. 2014. 
[5] L. Herda, P. Fua, R. Plänkers, R. Boulic, and D. Thalmann, "Using skeleton-based tracking to increase the reliability of optical motion capture," Hum. Movement Sci., vol. 20, no. 3, pp. 313-341, Jun. 2001.

[6] J. Hummel et al., "Evaluation of a new electromagnetic tracking system using a standardized assessment protocol," Phys. Med. Biol., vol. 51, no. 10, pp. N205-N210, May 2006.

[7] P. M. Mills, S. Morrison, D. G. Lloyd, and R. S. Barrett, "Repeatability of 3D gait kinematics obtained from an electromagnetic tracking system during treadmill locomotion," J. Biomech., vol. 40, no. 7, pp. 1504-1511, Jan. 2007.

[8] C. He, P. Kazanzides, H. Sen, S. Kim, and Y. Liu, "An inertial and optical sensor fusion approach for six degree-of-freedom pose estimation," Sensors, vol. 15, no. 7, pp. 16448-16465, Jul. 2015.

[9] H. Ren and P. Kazanzides, "Investigation of attitude tracking using an integrated inertial and magnetic navigation system for hand-held surgical instruments," IEEE/ASME Trans. Mechatronics, vol. 17, no. 2, pp. 210-217, Apr. 2012.

[10] X. Yun, J. Calusdian, E. R. Bachmann, and R. B. McGhee, "Estimation of human foot motion during normal walking using inertial and magnetic sensor measurements," IEEE Trans. Instrum. Meas., vol. 61, no. 7, pp. 2059-2072, Jul. 2012.

[11] M. Kok and T. B. Schon, "A fast and robust algorithm for orientation estimation using inertial sensors," IEEE Signal Process. Lett., vol. 26, no. 11, pp. 1673-1677, Nov. 2019

[12] Y. S. Suh, "Orientation estimation using a quaternion-based indirect Kalman filter with adaptive estimation of external acceleration," IEEE Trans. Instrum. Meas., vol. 59, no. 12, pp. 3296-3305, Dec. 2010.

[13] T. Seel, T. Schauer, and J. Raisch, "Joint axis and position estimation from inertial measurement data by exploiting kinematic constraints," in Proc. IEEE Int. Conf. Control Appl., Oct. 2012, pp. 45-49.

[14] H. G. Kortier, V. I. Sluiter, D. Roetenberg, and P. H. Veltink, "Assessment of hand kinematics using inertial and magnetic sensors," J. Neuroeng. Rehabil., vol. 11, no. 1, p. 70, 2014.

[15] J. Galka, M. Masior, M. Zaborski, and K. Barczewska, "Inertial motion sensing glove for sign language gesture acquisition and recognition," IEEE Sensors J., vol. 16, no. 16, pp. 6310-6316, Aug. 2016.

[16] B.-S. Lin, I.-J. Lee, S.-Y. Yang, Y.-C. Lo, J. Lee, and J.-L. Chen, "Design of an inertial-sensor-based data glove for hand function evaluation," Sensors, vol. 18, no. 5, p. 1545, May 2018.

[17] C. Salchow-Hömmen, L. Callies, D. Laidig, M. Valtin, T. Schauer, and T. Seel, "A tangible solution for hand motion tracking in clinical applications," Sensors, vol. 19, no. 1, p. 208, Jan. 2019.

[18] N. Enayati, E. De Momi, and G. Ferrigno, "A quaternion-based unscented Kalman filter for robust optical/inertial motion tracking in computer-assisted surgery," IEEE Trans. Instrum. Meas., vol. 64, no. 8, pp. 2291-2301, Aug. 2015.

[19] H. Ren, D. Rank, M. Merdes, J. Stallkamp, and P. Kazanzides, "Multisensor data fusion in an integrated tracking system for endoscopic surgery," IEEE Trans. Inf. Technol. Biomed., vol. 16, no. 1, pp. 106-111, Jan. 2012.

[20] S. Qiu, Z. Wang, H. Zhao, K. Qin, Z. Li, and H. Hu, "Inertial/magnetic sensors based pedestrian dead reckoning by means of multi-sensor fusion," Inf. Fusion, vol. 39, pp. 108-119, Jan. 2018.

[21] D. Weenk, D. Roetenberg, B.-J.-J. F. van Beijnum, H. J. Hermens, and P. H. Veltink, "Ambulatory estimation of relative foot positions by fusing ultrasound and inertial sensor data," IEEE Trans. Neural Syst. Rehabil. Eng., vol. 23, no. 5, pp. 817-826, Sep. 2015.

[22] D. Roetenberg, C. T. M. Baten, and P. H. Veltink, "Estimating body segment orientation by applying inertial and magnetic sensing near ferromagnetic materials," IEEE Trans. Neural Syst. Rehabil. Eng., vol. 15, no. 3, pp. 469-471, Sep. 2007.

[23] H. M. Schepers, D. Roetenberg, and P. H. Veltink, "Ambulatory human motion tracking by fusion of inertial and magnetic sensing with adaptive actuation," Med. Biol. Eng. Comput., vol. 48, no. 1, p. 27, Jan. 2010.

[24] H. G. Kortier, J. Antonsson, H. M. Schepers, F. Gustafsson, and P. H. Veltink, "Hand pose estimation by fusion of inertial and magnetic sensing aided by a permanent magnet," IEEE Trans. Neural Syst. Rehabil. Eng., vol. 23, no. 5, pp. 796-806, Sep. 2015.

[25] Z. Yang, B.-J. F. Van Beijnum, B. Li, S. Yan, and P. H. Veltink, "Estimation of relative hand-finger orientation using a small IMU configuration," Sensors, vol. 20, no. 14, p. 4008, 2020.

[26] A. Sheinker, B. Lerner, N. Salomonski, B. Ginzburg, L. Frumkis, and B.-Z. Kaplan, "Localization and magnetic moment estimation of a ferromagnetic target by simulated annealing," Meas. Sci. Technol., vol. 18 , p. 3451, Sep. 2007.
[27] Z. Yang, S. Yan, L. Chen, and B. Li, "Ferromagnetic object localization based on improved triangulating and ranging," IEEE Magn. Lett., vol. 10, no. 1, pp. 1-5, Apr. 2019.

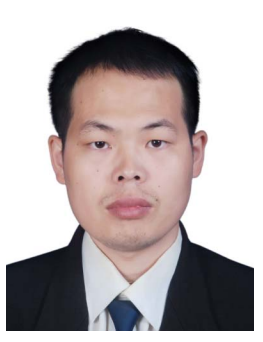

Zhicheng Yang received the B.Sc. degree in automation engineering from Zhengzhou University, Zhengzhou, China, in 2013. He is currently pursuing the Ph.D. degree with Northwestern Polytechnical University, Xi'an, China. In 2018 , he joined the University of Twente, Enschede, The Netherlands, as a joint Ph.D. Student. His main research interests include motion tracking with inertial sensors, inertial and magnetometer calibration, and localization of ferromagnetic object.

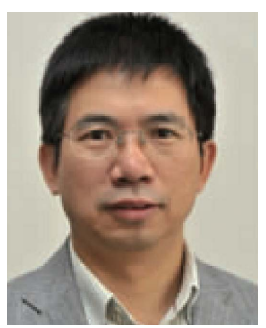

Shenggang Yan received the B.Sc. degree from Central South University, Changsha, China, in 1988, and the M.Sc. and Ph.D. degrees from Northwestern Polytechnical University, Xi'an, China, in 1991 and 2008, respectively. He is currently a Professor with Northwestern Polytechnical University. His main research interests include modern signal processing, high-speed signal processing, and their applications.

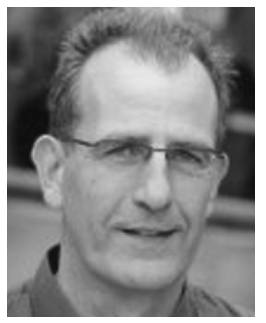

Bert-Jan F. van Beijnum (Member, IEEE) received the B.Sc. degree from Central South University, Changsha, China, in 1988, and the M.Sc. and Ph.D. degrees from Northwestern Polytechnical University, Xi'an, China, in 1991 and 2008, respectively. He is currently a Professor with Northwestern Polytechnical University. His main research interests include modern signal processing, high-speed signal processing, and their applications.

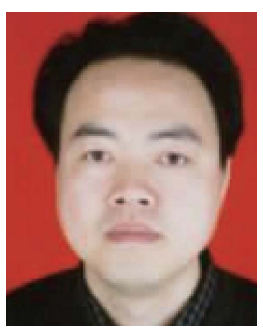

Bin Li received the B.Sc. and M.Sc. degrees from Northwestern Polytechnical University, Xi'an, China, in 1985 and 1988, respectively. He is currently a Professor with Northwestern Polytechnical University. His main research interests include magnetic target detection, sensor technology, and marine electromagnetic field communication.

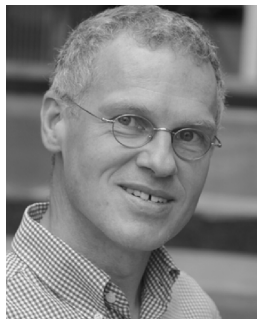

Peter H. Veltink (Senior Member, IEEE) is currently a Professor of technology for the restoration of human function with the University of Twente, Enschede, The Netherlands. $\mathrm{He}$ is the author or coauthor of over 125 peer-reviewed journal articles. He has been the scientific coordinator of three EU research training networks. $\mathrm{His}$ research interests include biomechatronics, neural engineering, ambulatory sensing of human movements, artificial human motor control, and neurostimulation. He was an Associate Editor of the IEEE TRANSACTIONS OF NEURAL SYSTEMS AND REHABILITATION ENGINEERING until 2014. 